Research Paper

\title{
Targeting P38 Pathway Regulates Bony Formation via MSC Recruitment during Mandibular Distraction Osteogenesis in Rats
}

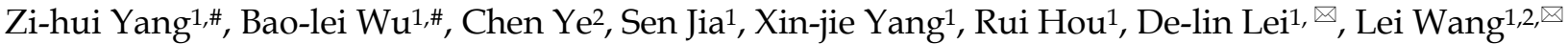 \\ 1. State Key Laboratory of Military Stomatology, Department of Oral and Maxillofacial Surgery, School of Stomatology, the Fourth Military Medical \\ University, China. \\ 2. Shanghai Key Laboratory of Stomatology, Department of Oral \& Maxillofacial-Head \& Neck Oncology, Ninth People's Hospital, School of Stomatology, \\ Shanghai Jiao Tong University School of Medicine, China \\ \# Joint first authors. \\ $\square$ Corresponding authors: Dr. Lei Wang MD, Ph.D at Key Laboratory of Stomatology, Department of Oral \& Maxillofacial-Head \& Neck Oncology, Ninth \\ People's Hospital, School of Stomatology, Shanghai Jiao Tong University School of Medicine, No.639 Zhizaoju Road, Shanghai 200011, China. Email: \\ wangleizyh@aliyun.com, Phone: +86 21 63166731; Fax: +86 21 63166731; or Prof. De-lin Lei MD at State Key Laboratory of Military Stomatology, Department of \\ Oral and Maxillofacial Surgery, School of Stomatology, the Fourth Military Medical University, No.145 West Changle Road, Xi'an 710032, China. Email: \\ leidelin@fmmu.edu.cn, Phone: +86 29 84772501; Fax: +86 84776011.
}

(0) Ivyspring International Publisher. Reproduction is permitted for personal, noncommercial use, provided that the article is in whole, unmodified, and properly cited. See http://ivyspring.com/terms for terms and conditions.

Received: 2016.06.30; Accepted: 2016.09.01; Published: 2016.10.01

\begin{abstract}
Distraction osteogenesis (DO) is a widely used self-tissue engineering. However, complications and discomfort due to the long treatment period are still the bottleneck of DO. Novel strategies to accelerate bone formation in DO are still needed. P38 is capable of regulating the osteogenic differentiation of both mesenchymal stem cells (MSCs) and osteoblasts, which are crucial to bone regeneration. However, it is not clear whether targeting $\mathrm{p} 38$ could regulate bony formation in DO. The purpose of the current work was to investigate the effects of local application of either $\mathrm{p} 38$ agonist anisomycin or p38 inhibitor SB203580 in a rat model of DO. 30 adult rats were randomly divided into 3 groups: (A) rats injected with DMSO served as the control group; (B) rats injected with p38 agonist anisomycin; (C) rats injected with p38 inhibitor SB203580. All the rats were subjected to mandibular distraction and the injection was performed daily during this period. The distracted mandibles were harvested on days 15 and 30 after surgery and subjected to the following analysis. Micro-computed tomography and histological evaluation results showed that local application of p38 agonist anisomycin increased new bone formation in DO, whereas p38 inhibitor SB203580 decreased it. Immunohistochemical analysis suggested that anisomycin promoted MSC recruitment in the distraction gap. In conclusion, this study demonstrated that local application of $\mathrm{p} 38$ agonist anisomycin can increase new bone formation during DO. This study may lead to a novel cell-based strategy for the improvement of bone regeneration.
\end{abstract}

Key words: distraction osteogenesis, mesenchymal stem cell, mandible, p38 signaling, anisomycin.

\section{Introduction}

Distraction osteogenesis (DO) is a widely used tissue engineering technique in bone repair. However, complications and discomfort due to the long treatment period are still the bottleneck of DO [1,2]. Despite the existence of several studies on accelerating DO [3-5], novel stem-cell-based strategies to accelerate bone formation and promote the therapeutic effect of DO are still needed.

MSCs play a pivotal role in bone regeneration $[6,7]$, which is regulated by series of signals triggered by loaded strain during DO ${ }^{[8]}$. P38 is an important member of the mitogen-activated protein kinase superfamily (MAPK) [9]. It is capable of regulating such processes as cell proliferation and mechano- 
transduction [10]. Recent studies demonstrated that p38 is capable of regulating osteogenesis of MSCs and MSC-derived osteoblasts. Kwon et al. [11] demonstrated that p38 signaling provides a regulatory control of myocilin-induced osteogenic differentiation of MSCs. Thouverey et al. [12] found that osteoblast-specific p38a knock-out mice showed significant decreases in bone mineral density. However, the role of p38 in the mediation of osteogenesis in DO and whether targeting p38 signaling can stimulate bone regeneration during DO are still unknown.

In the present study, a rat model of mandibular $\mathrm{DO}$ was used. Both microCT and histological analysis were performed to evaluate the effect of anisomycin and SB203580 application on DO. Immunohistochemical analysis was used to assess the contributions of MSCs in the use of anisomycin during DO. To the best of our knowledge, this study is the first to investigate the effect of targeting p38 signaling on DO.

\section{Materials and Methods}

\section{Animal grouping and surgical protocol}

Thirty male SD rats weighing $290 \pm 10.5 \mathrm{~g}$ were randomly divided into 3 groups. (A) Rats $(n=10)$ were injected with $200 \mu \mathrm{L}$ DMSO served as the control; (B) rats $(n=10)$ were injected with anisomycin at a dose of $2.5 \mathrm{mg} / \mathrm{kg}$ in $200 \mu \mathrm{L}$ DMSO; (C) rats $(n=10)$ were injected with SB203580, a p38 inhibitor, at a dose of $2.5 \mathrm{mg} / \mathrm{kg}$ in $200 \mu \mathrm{L}$ DMSO. All the animals were subjected to mandibular distraction osteogenesis as described in a previous study [13]. Briefly, rats were injected intraperitoneally with $1 \%$ pentobarbital sodium. After the rats were anaesthetized, a vertical osteotomy was created in the retromolar area and a custom-made distraction device was placed and fixed with 2 screws on each side. After 5 days of latency, the mandibles were distracted at a rate of $0.2 \mathrm{~mm} / 12 \mathrm{~h}$ for 10 days. The injections were performed during this distraction period. Five rats in each group were killed on day 15 and another five on day 30 post surgery. Mandibles were harvested and subjected to the following analysis.

\section{Micro-CT evaluation}

The mandibles harvested were examined using a micro-CT system (Inveon CT, Siemens AG, Munich, Germany). The protocol was described in a previous study [14]. Briefly, each mandible was scanned, and about 1000 pictures were taken, each at a resolution of $1888 \times 2048$ pixels in an isotropic size of $15 \mu \mathrm{m}$. The analysis included bone mineral density (BMD) and bone volume/total volume (BV/TV). All evaluations were conducted in triplicate.

\section{Histological and immunohistochemistry evaluation}

After microCT scanning, specimens were processed for histological analysis. The mandibles were fixed with $4 \%$ paraformaldehyde at $4^{\circ} \mathrm{C}$ for $48 \mathrm{~h}$, decalcified in $10 \%$ EDTA for 4 weeks, embedded in paraffin, and sliced in $5 \mu \mathrm{m}$ sections along the axial plane. The slices were subjected to hematoxylin-eosin and immunohistochemical staining.

For hematoxylin-eosin staining, images were taken in 5 randomly selected high magnification fields (200x) per slide under a microscope. The new bone formation in the distraction gap was quantified with the ratio of trabecular bone volume/total volume using Image Pro-Plus analysis software (Media Cybernetics, Inc., Rockville, MD, U.S.) by an experienced pathologist.

For immunohistochemical staining, the tissue sections were incubated with anti-Nestin (1:100, Abcam, U.S.). The brown particles in the cytoplasm were considered positive. Images were photographed in 5 randomly selected fields $(400 \times)$ per slide. The Nestin $^{+}$cells were counted manually by an experienced pathologist. All the experiments were conducted in triplicate.

\section{Statistical analysis}

Statistics were measured with the SPSS 17.0 software (IBM, Armonk, NY, U.S.). Data were presented as the mean \pm SEM. The significance was evaluated using one-way analysis of variance (ANOVA). $P<0.05$ was considered as significant.

\section{Results}

All the animals tolerated the surgery well and were subjected to the following analysis.

\section{Gross view of the specimens}

As shown in Figure 2, all the mandibles were successfully lengthened. On day 15 , the broken ends were obvious, and the distraction gap was filled with soft tissues and a few calcified tissues in each group. All the mandibles were able to be twisted somewhat. On day 30, the broken ends were still visible in the control and the SB203580 group, whereas indistinct in the anisomycin group. The gaps were filled with the calcified tissues and not able to be twisted in the anisomycin or control group, whereas a great deal of soft tissues was still visible and able to be twisted in the SB203580 group.

\section{Micro-CT evaluation}

As shown in Figure 3, bone callus was visible in the distraction gap of each group. On day 15, most areas of the gap were radiolucent, with the bone callus 
close to the proximal or distal end in the control and SB203580 group, whereas the gap in the anisomycin group was largely filled with bone callus. On day 30, the volume of bone callus increased a lot than that on day 15. The broken ends in the control and anisomycin group were still clear, but they were obscure in the anisomycin group. Both the BV/TV ratio and BMD value in the gap of anisomycin group were significantly higher than those of the control $(P<0.05)$, but they were lower in the SB203580 group than in the control at any point in time $(P<0.05)$. These data suggested that the anisomycin application promoted bony formation in DO.

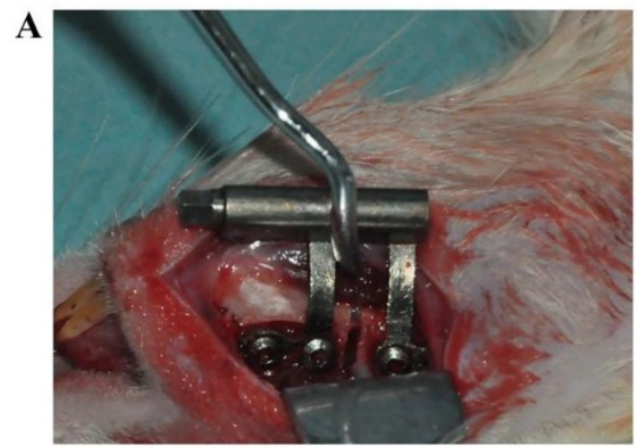

\section{Histology and histomorphometry analysis}

On day 15, the bundles of fibers oriented along the distraction axis with calcified tissue and osteoid matrix deposited in the distraction gaps of each group. They were surrounded by dense cells and abundant blood vessels without any sign of inflammation. On day 30, the trabecular bone volume increased, aligning along the axis of distraction. The trabeculae were separated from each other in the control and SB203580 group but they were fused in the anisomycin group, whose structure was more similar to that of the mature lamellar bone (shown in Figure 4A).
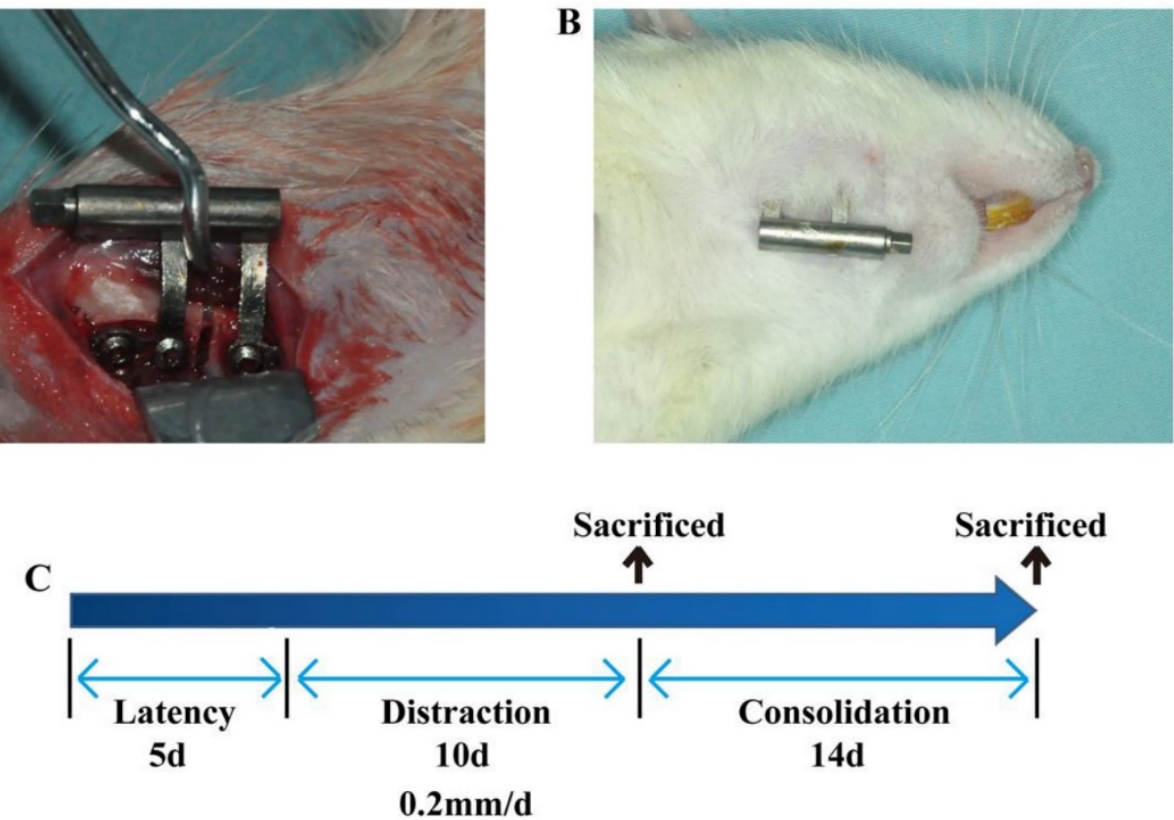

Figure 1. The rat model of mandibular DO used in this study. (A) Distractor implantation. (B) Rats were killed, and the incision area was clean without sign of inflammation. (C) Schematic diagram of DO. After 5 days of latency, the right mandible of the rat was distracted at a rate of $0.2 \mathrm{~mm} / 12 \mathrm{~h}$ for 10 days. Rats in this study were sacrificed on day 15 (five rats in each group) or on day 30 (five rats in each group) after 14 days of consolidation.

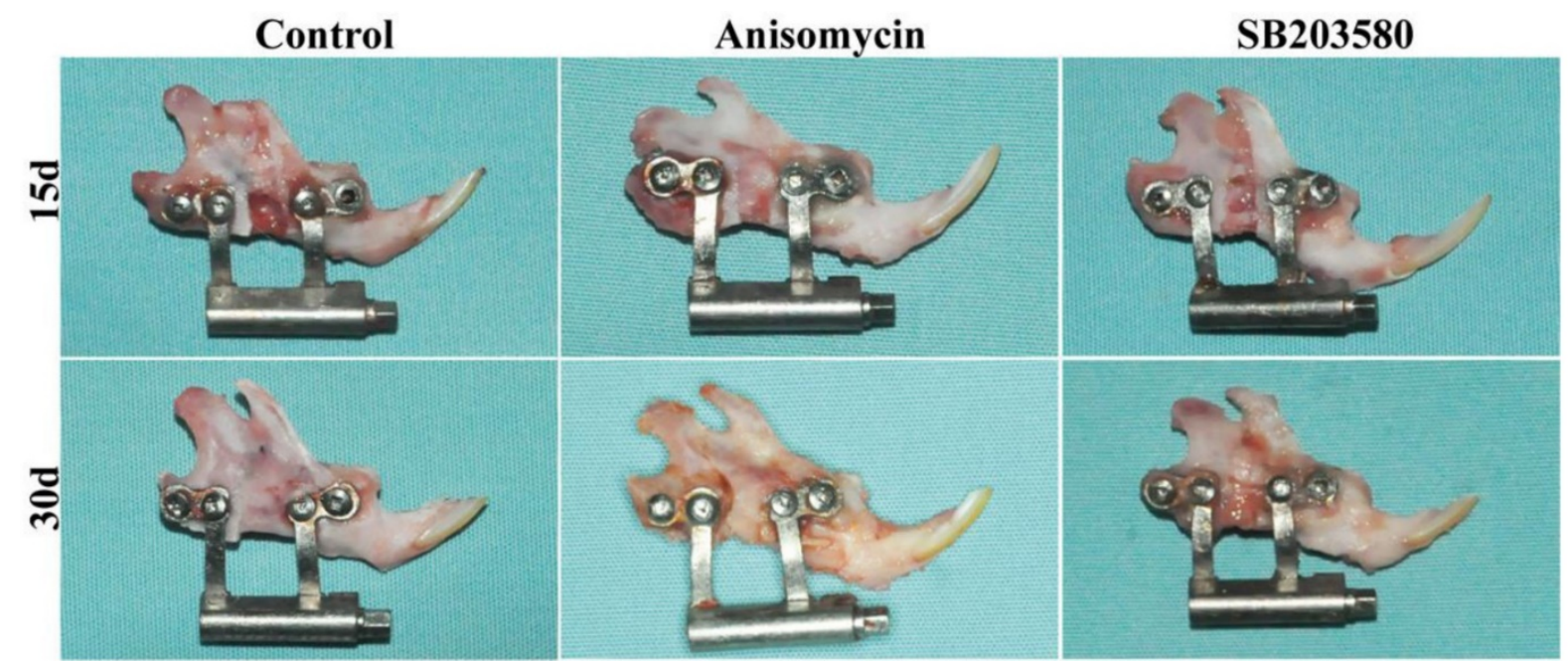

Figure 2. Gross view of mandibles on day 15 and 30 post surgery. Distracted mandibles were harvested on day 15 and 30 respectively. No sign of inflammation was shown in each group. 
A

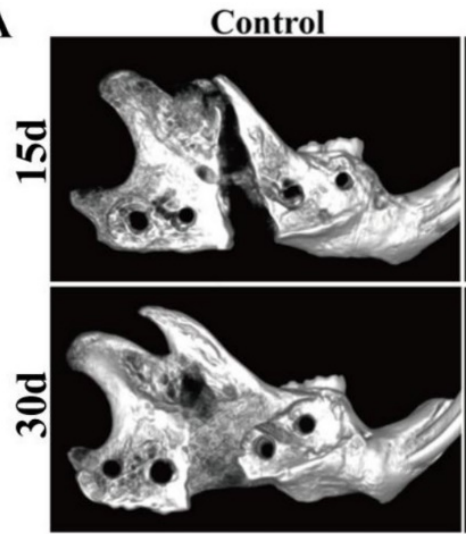

B

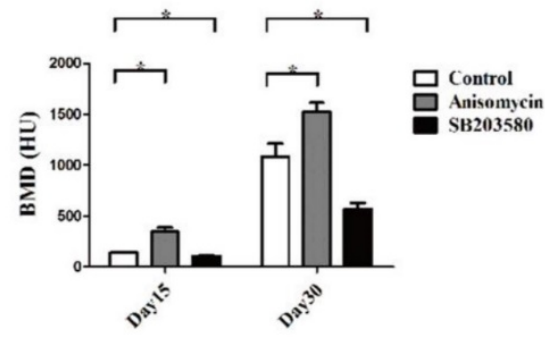

Anisomvcin
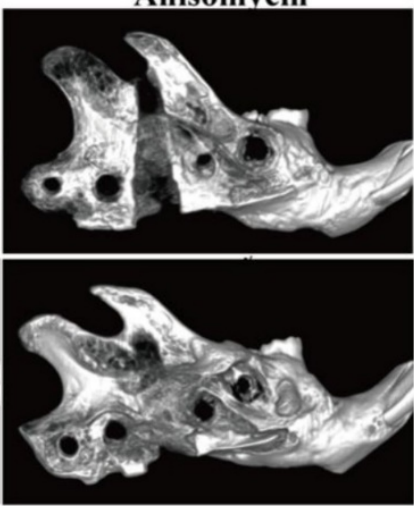

SB203580

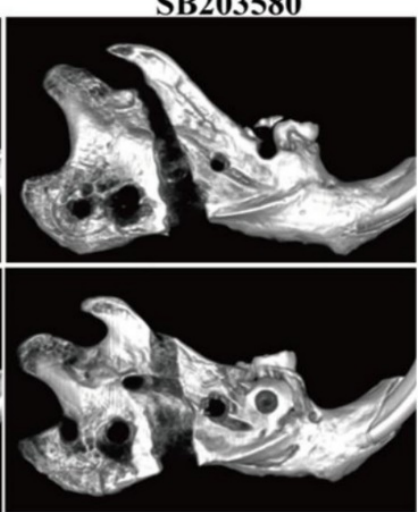

C

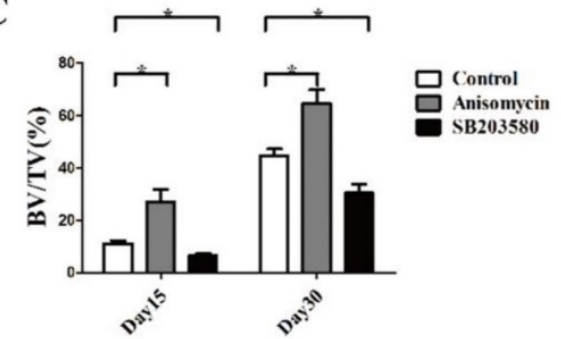

Figure 3. MicroCT evaluation of each group. (A) Images of mandibles (B) Bone mineral density (BMD) analysis. (C) The ratio of bone volume/total volume (BV/TV) analysis.

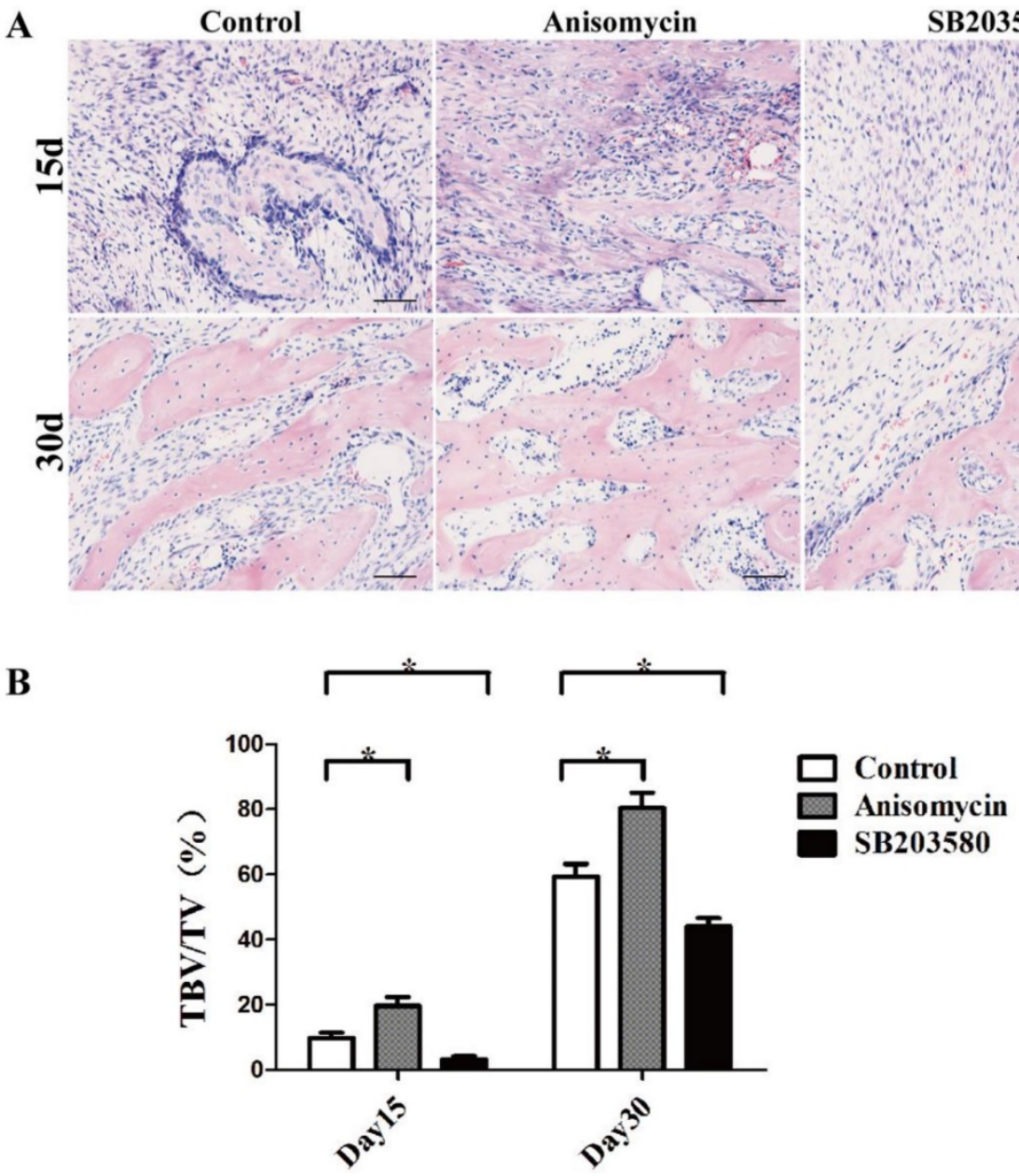

Figure 4. Histology and histomorphometric analysis of each group. (A) HE photographs of day 15 and day 30 . (B) Trabecular bone volume/ total volume analysis. Images were taken in 5 randomly selected high magnification fields $(200 \times)$ per slide under a microscope. The new bone formation in the distraction gap was quantified with the ratio of trabecular bone volume/total volume using Image Pro-Plus analysis software by an experienced pathologist. Bar $=50 \mu \mathrm{m}$. 


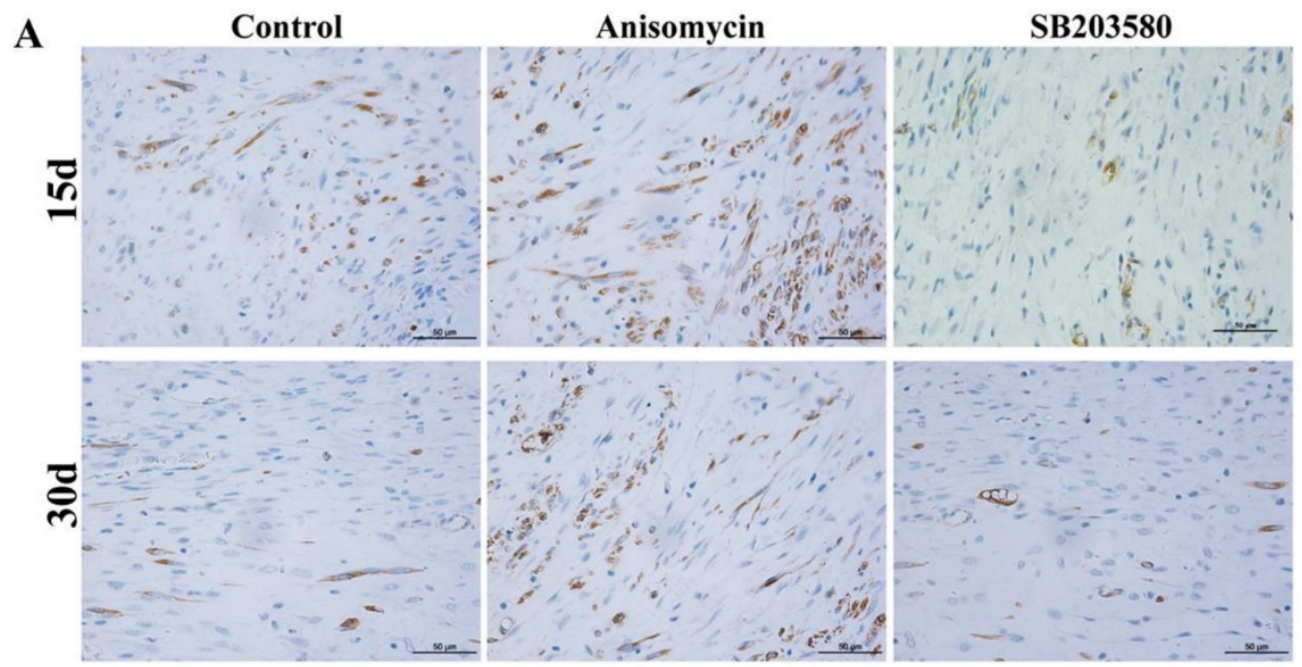

B

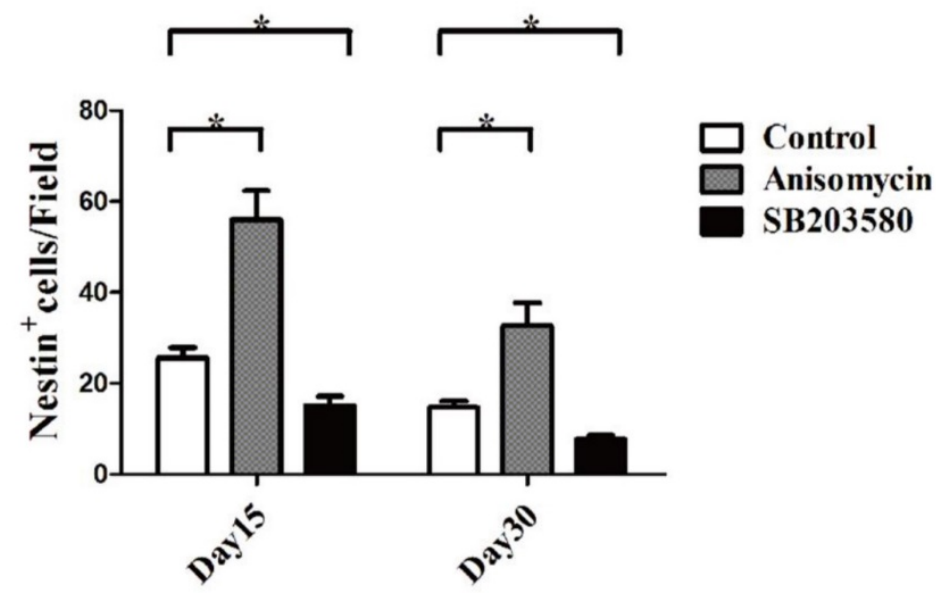

Figure 5. Nestin immunohistochemistry analysis of each group. (A) Nestin staining photographs of each group on day 15 and 30 . (B) Nestin + cell analysis. Images were photographed in 5 randomly selected fields $(400 \times)$ per slide. The Nestin+ cells were counted manually by an experienced pathologist. All the experiments were conducted in triplicate. Bar $=50 \mu \mathrm{m}$.

Trabecular bone tissue volume/total volume (TBV/TV) analysis was used here to evaluate the new bone formation in each group. The results showed that both on day 15 and day 30, there was significantly more newly formed trabecular bone in the anisomycin group than in the control group $(P<0.05)$, but there was less in the SB203580 group than in the control group $(P<0.05)$ (shown in Figure $4 \mathrm{~B})$. These data were in accordance with the microCT evaluation, demonstrating that the use of anisomycin promoted bony formation in DO.

\section{Nestin immunohistochemistry evaluation}

Next, Nestin immunohistochemistry assays were performed. Nestin was used here as a MSCs marker. As shown in Figure 5, MSCs were able to be detected in the distraction gap at each point in time. There were fewer MSCs on day 30. On both day 15 and day 30, the numbers of MSCs in the anisomycin group were significantly higher than that in the control group $(P<0.05)$, but they were significantly lower in the SB203580 group than in the control group $(P<0.05)$.

\section{Discussion}

DO is a potent bioactivator that induces new bone formation through continuous strain loaded on each broken end. The conversion of the strain signals to intracellular molecular signaling, which is known as the process of mechanotransduction [15], is vital to bone regeneration in DO. P38 has been shown to be capable of mediating the process of mechanotransduction in MSCs [8]. It is well documented that p38 exerts its regulatory control over the phenotypic fate of MSCs, and the activation of p38 signaling favors MSCs into osteoblast linages [11, 16]. MSCs have been shown to reside in a dedicated microenvironment known as the stem cell niche, in which self-renewal is maintained and differentiation 
inhibited [17,18]. MSCs are sensitive to alterations in the stress microenvironment, and it is well documented that mechanical strain, both static and cyclic, promotes the osteogenesis of MSCs, which is critical to bone regeneration in DO [19,20]. However, it is still not clear whether targeting p38 signaling can increase new bone formation during DO. Here, the effect of anisomycin, a p38 agonist, has been investigated in a rat mandibular DO model which was demonstrated to be useful and reproducible in vivo model for DO in previous studies [13, 14, 21]. The results showed that locally injected anisomycin can increase bone formation in DO. This study is the first report to show that the local application of anisomycin can promote bone regeneration in $\mathrm{DO}$.

Yasui et al. [22] carried out a study on the ossification process in a rat model of limb lengthening. The results showed there to be three ossification modes during DO: endochondral, intramembranous, and transchondroid ossification. The endochondral and transchondroid ossification were mainly visible during the first 10 days of distraction. Afterwards, intramembranous ossification prevailed. The latest studies have reported bone regeneration in DO to be mainly the result of intramembranous ossification, unlike fracture healing [23]. The present study showed osteoid tissue deposited in the distraction gap on day 15 in each group, surrounded by abundant vessels and fibroblast-like cells. Immature trabeculae were visible in the anisomycin and control groups. On day 30, a large mass of trabecular bone formed in the distraction gap of each group. The trabeculae fused in the anisomycin group, with a sign of bone remodeling. There was almost no cartilage or chondroid in any groups. These results suggested that the bone formation in all the three groups took place mainly through intramembranous ossification. These findings were consistent with those of previous studies. The microCT and histological analysis showed that local application of anisomycin resulted in a significantly higher bony volume than that of the control, whereas application of SB203580 led to a less bone formation than the control treatment at each point in time. It was here concluded that local injection of anisomycin can considerably increase intramembranous ossification during DO.

MSCs have been demonstrated to be essential to bone regeneration in DO. Nestin immunohistochemistry was performed to assess the contributions of MSCs in each group. Nesin here served as a MSCs marker. The results showed there to be significantly more MSCs in the gaps of the anisomycin group than in the control group at each point in time, but there were fewer in the SB203580 group. The increase in the number of MSCs may account for the accelerated bone formation in the anisomycin group. This suggested that there may be more MSCs differentiating towards osteoblast linages. It has been reported that p38 is capable of mediating stromal cell-derived factor-1/CXCR4 (SDF-1/CXCR4) axis in MSCs [24]. The interaction of SDF-1 with CXCR4 plays a crucial regulatory role in MSCs recruitment [25]. It was here considered that the anisomycin-induced MSC accumulation in this study may be partly due to the enhanced recruitment of MSCs. There were still more MSCs in the anisomycin group than in the control group on day 30, in which the injection was terminated, suggesting that other factors may be involved in the regulation of MSCs. Taken together, these data indicated that the increased bone regeneration in the anisomycin group may not only be due to the enhanced differentiation of MSCs but may also be caused by the accelerated recruitment of MSCs. However, the exact mechanism has been elucidated and further investigations are needed.

\section{Conclusions}

In conclusion, it is here demonstrated that local application of p38 agonist anisomycin can increase new bone formation during DO. This study may lead to the development of a novel cell-based strategy to improve bone regeneration.

\section{Abbreviations}

DO: distraction osteogenesis; MSCs: mesenchymal stem cells; Micro-CT: micro-computed tomography; BV/TV: bone volume/total volume; BMD: bone mineral density; TBV/TV: trabecular bone tissue volume/total volume; DMSO: dimethylsulfoxide; MAPK: mitogen-activated protein kinase superfamily.

\section{Acknowledgements}

We thank LetPub (www.letpub.com) for its linguistic assistance during the preparation of this manuscript. This work was supported by the National Natural Science Foundation of China (No. 81270015 to L.W and No. 81070811 to D.L.L).

\section{Competing Interests}

The authors have declared that no competing interest exists.

\section{References}

1. Donneys A, Deshpande SS, Tchanque-Fossuo $\mathrm{CN}$, et al. Deferoxamine expedites consolidation during mandibular distraction osteogenesis. Bone. 2013; 55: 384-90.

2. Nomura I, Watanabe $\mathrm{K}$, Matsubara $\mathrm{H}$, et al. Uncultured autogenous adipose-derived regenerative cells promote bone formation during distraction osteogenesis in rats. Clin Orthop Relat Res. 2014; 472: 3798-806. 
3. Li W, Zhu S, Hu J. Bone Regeneration Is Promoted by Orally Administered Bovine Lactoferrin in a Rabbit Tibial Distraction Osteogenesis Model. Clin Orthop Relat Res. 2015; 473: 2383-93.

4. Wang $X$, Zhu S, Jiang $X$, et al. Systemic administration of lithium improves distracted bone regeneration in rats. Calcif Tissue Int. 2015; 96: 534-40.

5. Jiang $X$, Zhang $Y$, Fan $X$, et al. The effects of hypoxia-inducible factor (HIF)-1a protein on bone regeneration during distraction osteogenesis: an animal study. Int J Oral Maxillofac Surg. 2016; 45: 267-72.

6. Bodle JC, Loboa EG. Concise Review: Primary Cilia: Control Centers for Stem Cell Lineage Specification and Potential Targets for Cell-Based Therapies. Stem Cells. 2016; 34: 1445-54.

7. Dawson JI, Kanczler J, Tare R, et al. Concise review: bridging the gap: bone regeneration using skeletal stem cell-based strategies - where are we now. Stem Cells. 2014; 32: 35-44

8. Kim IS, Song YM, Hwang SJ. Osteogenic responses of human mesenchymal stromal cells to static stretch. J Dent Res. 2010; 89: 1129-34.

9. Chang L, Karin M. Mammalian MAP kinase signalling cascades. Nature. 2001; 410: $37-40$.

10. Kim EK, Choi EJ. Compromised MAPK signaling in human diseases: an update. Arch Toxicol. 2015; 89: 867-82

11. Kwon HS, Johnson TV, Tomarev SI. Myocilin stimulates osteogenic differentiation of mesenchymal stem cells through mitogen-activated protein kinase signaling. J Biol Chem. 2013; 288: 16882-94.

12. Thouverey C, Caverzasio J. The p38a MAPK positively regulates osteoblast function and postnatal bone acquisition. Cell Mol Life Sci. 2012; 69: 3115-25.

13. Cao J, Wang L, Du ZJ, et al. Recruitment of exogenous mesenchymal stem cells in mandibular distraction osteogenesis by the stromal cell-derived factor-1/chemokine receptor-4 pathway in rats. Br J Oral Maxillofac Surg. 2013; 51: 937-41.

14. Zhang $\mathrm{YB}$, Wang $\mathrm{L}$, Jia $\mathrm{S}$, et al. Local injection of substance $\mathrm{P}$ increases bony formation during mandibular distraction osteogenesis in rats. Br J Oral Maxillofac Surg. 2014; 52: 697-702.

15. Papachristou DJ, Papachroni KK, Basdra EK, Papavassiliou AG. Signaling networks and transcription factors regulating mechanotransduction in bone. Bioessays. 2009; 31: 794-804.

16. $\mathrm{Pu} \mathrm{Y}, \mathrm{Wu} \mathrm{H}, \mathrm{Lu} \mathrm{S}$, et al. Adiponectin Promotes Human Jaw Bone Marrow Stem Cell Osteogenesis. J Dent Res. 2016; 95: 769-75.

17. Mendez-Ferrer S, Michurina TV, Ferraro F, et al. Mesenchymal and haematopoietic stem cells form a unique bone marrow niche. Nature. 2010; 466: 829-34.

18. Kfoury Y, Scadden DT. Mesenchymal cell contributions to the stem cell niche. Cell Stem Cell. 2015; 16: 239-53.

19. Sen $\mathrm{B}, \mathrm{Xie} Z$, Case N, et al. mTORC2 regulates mechanically induced cytoskeletal reorganization and lineage selection in marrow-derived mesenchymal stem cells. J Bone Miner Res. 2014; 29: 78-89.

20. Luu YK, Capilla E, Rosen CJ, et al. Mechanical stimulation of mesenchymal stem cell proliferation and differentiation promotes osteogenesis while preventing dietary-induced obesity. J Bone Miner Res. 2009; 24: 50-61.

21. Du Z, Wang L, Zhao $\mathrm{Y}$, et al. Sympathetic denervation-induced MSC mobilization in distraction osteogenesis associates with inhibition of MSC migration and osteogenesis by norepinephrine/adrb3. PLoS One 2014; [Epub ahead of print].

22. Yasui $\mathrm{N}$, Sato $\mathrm{M}$, Ochi $\mathrm{T}$, et al. Three modes of ossification during distraction osteogenesis in the rat. J Bone Joint Surg Br. 1997; 79: 824-30.

23. Dhaliwal K, Kunchur R, Farhadieh R. Review of the cellular and biological principles of distraction osteogenesis: An in vivo bioreactor tissue engineering model. J Plast Reconstr Aesthet Surg 2016; [Epub ahead of print].

24. Ryu CH, Park SA, Kim SM, et al. Migration of human umbilical cord blood mesenchymal stem cells mediated by stromal cell-derived factor-1/CXCR4 axis via Akt, ERK, and p38 signal transduction pathways. Biochem Biophys Res Commun. 2010; 398: 105-10

25. Hwang JH, Kim SW, Park SE, et al. Overexpression of stromal cell-derived factor-1 enhances endothelium-supported transmigration, maintenance, and proliferation of hematopoietic progenitor cells. Stem Cells Dev. 2006; 15: 260-8. 